TITLE:

\title{
Live-cell imaging of endogenous mRNAs with a small molecule.
}

\section{$\operatorname{AUTHOR}(\mathrm{S})$ :}

Sato, Shin-ichi; Watanabe, Mizuki; Katsuda, Yousuke; Murata, Asako; Wang, Dan Ohtan; Uesugi, Motonari

\section{CITATION:}

Sato, Shin-ichi ... [et al]. Live-cell imaging of endogenous mRNAs with a small molecule.. Angewandte Chemie 2014, 54(6): 1855-1858

\section{ISSUE DATE:}

2014-12-23

URL:

http://hdl.handle.net/2433/198615

\section{RIGHT:}

This is the peer reviewed version of the following article: Sato, S.-i., Watanabe, M., Katsuda, Y., Murata, A., Wang, D. O. and Uesugi, M. (2015), Live-Cell Imaging of Endogenous mRNAs with a Small Molecule. Angew. Chem. Int. Ed., 54:

1855-1858, which has been published in final form at http://dx.doi.org/10.1002/anie.201410339.; 許諾条件により本文フ アイルは2015-12-23に公開.; This is not the published version. Please cite only the published version.; この論文は出版社 版でありません。引用の際には出版社版をご確認ざ利用ください。 


\title{
Live-Cell Imaging of Endogenous mRNAs with a Small Molecule**
}

\author{
Shin-ichi Sato,* Mizuki Watanabe, Yousuke Katsuda, Asako Murata, Dan Ohtan Wang, and Motonari Uesugi*
}

\begin{abstract}
Determination of subcellular localization and dynamics of mRNA is increasingly important to understanding gene expression. We report a new convenient and versatile method that permits spatiotemporal imaging of specific non-engineered RNAs in living cells. The method uses transfection of a plasmid encoding a gene-specific RNA aptamer, combined with a cell-permeable synthetic small molecule whose fluorescence is restored only when the RNA aptamer hybridizes with its cognitive mRNA. The method was validated by live-cell imaging of the endogenous mRNA of $\beta$-actin. Application of the technology to mRNAs of a total of 84 human cytoskeletal genes allowed us to observe cellular dynamics of several endogenous mRNAs including ARFIP2, CTTN, and CYFIP2. The RNA-imaging technology and its further optimization might permit live-cell imaging of any RNA molecules.
\end{abstract}

Spatiotemporal regulation of gene expression in living cells is controlled by the stability and transport of mRNA, in addition to gene transcription and regulated protein synthesis and degradation. Although intracellular localization of RNA has been determined by in situ hybridization in fixed and permeabilized cells ${ }^{[1]}$, this method provides only a snapshot image at the time of fixation. In contrast, live-cell imaging allows examination of both distribution and dynamics of mRNAs in cells, and provides new perspectives into the cell biology of mRNA ${ }^{[2]}$.

An increasing number of methods for live-cell RNA imaging have been developed ${ }^{[3]}$. In the MS2 and its orthogonal systems, a tandem array of repetitive RNA tag is fused to the gene of interest, which can be specifically recognized and bound with high affinity by an RNA-binding protein-green fluorescent protein (GFP) fusion proteins expressed simultaneously ${ }^{[4]}$. In a more recently developed "spinach system," an engineered RNA aptamer tag switches on the fluorescence of GFP-like fluorophores ${ }^{[5]}$. Although both methods allow visualization of RNA transcripts in living cells, their use is limited to engineered, non-native mRNA.

A method that is applicable to non-engineered, endogenous mRNAs in living cells would be extremely useful, and several have been developed. One approach uses molecular beacons, whose fluorescence changes upon binding to native mRNA targets ${ }^{[6]}$. The high designability of this pioneering method for given RNA targets permitted visualization of a wide range of endogenous RNAs in living cells ${ }^{[7]}$. However several

[*] Dr. S. Sato, Dr. M. Watanabe, Dr. Y. Katsuda, Dr. A. Murata, Dr. DO. Wang, Prof. M. Uesugi Institute for Integrated Cell-Material Sciences (WPI-iCeMS) and Institute for Chemical Research, Kyoto University, Uji, Kyoto 611-0011, Japan E-mail: ssato@scl.kyoto-u.ac.jp and uesugi@scl.kyoto-u.ac.jp

[**] We thank C. Manabe, N.M. Mbenza, M. Takeuchi, J. Takaya, G. Jin, and I. Oomoto for their technical assistance. This work was supported in part by JSPS (26220206 to M.U. and 23510277 to S.S.), JST (AS242Z01268P to S.S), ZE Research Program, IAE (B-7 to S.S.), and an iCeMS research acceleration grant. iCeMS is supported by World Premier International Research Center Initiative (WPI), MEXT, Japan. This work was inspired by the international and interdisciplinary environments of the iCeMS and JSPS Asian CORE Program, “Asian Chemical Biology Initiative.” The upgrade of the con-focal microscope was supported by the New Energy and Industrial Technology Development Organization (NEDO) of Japan and Yokogawa Electric Corporation. The NMR spectra were recorded on the NMR spectrometers in the JURC at Institute for Chemical Research, Kyoto University. challenges remain: for example, low stability of the beacons in cells produces relatively high background fluorescence. Such drawbacks in molecular beacons have recently been overcome by taking advantages of turn-on probes. For instance, locked nucleotide-enhanced DNAforced-intercalation probes (LNT-enhanced FIT probes) promote their fluorescence only upon hybridization with their cognitive RNAs ${ }^{[8]}$. High signal-to-noise ratio and nuclease resistance of the LNT-enhanced FIT probes allowed detection of the dynamics of oskar mRNA in Drosophila melanogaster oocytes. Another recently developed approach uses hybrid proteins that combine split GFP and RNA-binding proteins ${ }^{[9]}$. This genetically encoded approach has provided valuable information about the dynamics of endogenous RNAs, including mitochondrial RNA encoding NADH dehydrogenase subunit 6 . Unfortunately, systematic use of this method has been hampered by the need to design a specific probe for each RNA target. Thus, development of a versatile and robust technique for visualizing endogenous RNA in living cells is of great interest.

We previously described an RNA imaging method in which the fluorescence of a quencher-fluorophore conjugate, BHQ-sulfo-Cy3, is restored in the presence of an RNA aptamer that binds to the quencher $\mathrm{BHQ}^{[10]}$. Application of this method to non-engineered mRNA in living cells was hampered by the lack of the RNA aptamer sequence in endogenous mRNAs, and by low cell permeability of BHQ-sulfo-Cy3 (Figure S1). To improve cell permeability, we removed its acidic sulfate groups. Fluorescence microscopy showed that the resulting BHQfluorophore, conjugate $\mathbf{1}$ (Figure 1A), diffused throughout the cytoplasm in HeLa cells (Figures S1 and S2). Therefore, conjugate $\mathbf{1}$ was used for further studies.

We exploited the stem-loop structure of the original BHQ-binding RNA aptamer ${ }^{[10]}$, which is essential for BHQ recognition, to make it responsive to sequences of specific native mRNA (Figure 1B). Disruption of the stem formation destabilizes the BHQ-recognition loop, leading to a loss of BHQ-binding activity ${ }^{[10]}$. This highly sensitive structural property allowed us to design an RNA aptamer responsive to the sequence of an endogenous RNA. The original stem segment of the BHQ aptamer was replaced with two short RNA sequences complementary to a 24-base oligonucleotide in a target RNA. The sequences connect with a 3-base-pair stem segment through $\mathrm{U}$ mononucleotide bridges (Figure S3). The resulting RNA-targeting aptamer (RT-aptamer) can only form a BHQ-recognition loop by hybridizing with its complementary RNA. Thus, an RT-aptamer induced a five-fold increase in fluorescence in conjugate $\mathbf{1}$ in the presence of its cognitive RNA, whereas conjugate $\mathbf{1}$ was unresponsive to the RTaptamer alone (Figure 1C). The RT-aptamer and target RNA enhanced the fluorescence of conjugate $\mathbf{1}$ in a dose-dependent manner (Figure S4).

Strict sequence-selectivity is essential for live-cell imaging of a specific RNA. To assess selectivity, we examined whether the RTaptamer discriminates three partially complementary RNA oligonucleotides that cannot stabilize the stem-loop structure of the RTaptamer (Figure S5). In contrast to the fully complementary RNA oligonucleotide, none of the partially complementary oligonucleotides with the RT-aptamer restored the fluorescence of conjugate $\mathbf{1}$. We also examined the effects of point mutations of the complementary RNA oligonucleotide on selectivity (Figure S6). The fluorescence signal of conjugate $\mathbf{1}$ was highly responsive to single nucleotide changes, 
particularly in the middle of the cognitive sequence, even though the RTaptamer hybridized with the mutants along the rest of 19-base cognitive sequence. These results suggest that in vitro formation of the stem-loop structure of the RT-aptamer, which is essential for BHQ recognition, is highly dependent on the sequence of the target RNA.

A

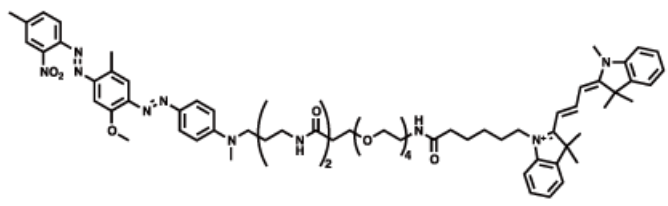

BHQ-fluorophore conjugate 1
B

(a)

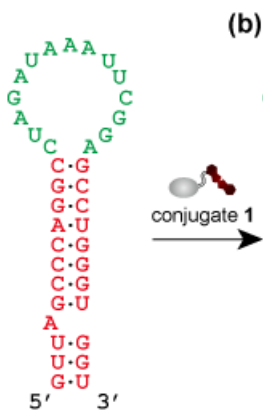

(d)

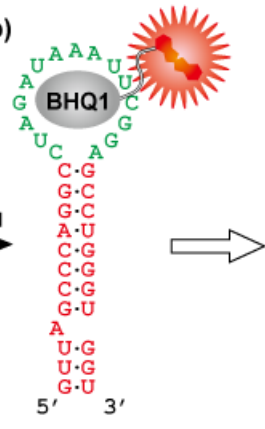

(c)

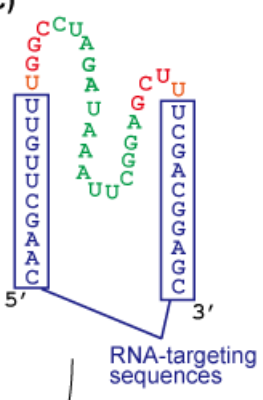

Fluorescence intensities of conjugate 1 (final concentration: $5 \mu \mathrm{M}$ ) with the RTaptamer $(10 \mu \mathrm{M})$ in the presence or absence of a 15- $\mu \mathrm{M}$ target RNA (red and blue, respectively), and without the RT-aptamer (black). The RT-aptamer increases the fluorescence intensities of conjugate 1 upon hybridization with its target RNA.

To determine whether RT-aptamers are capable of targeting internal sequences of a long mRNA in vitro, we used two mRNAs encoding GFP, in which 3'-UTR was either present or absent (Figure S7). In the presence of an RT-aptamer complementary to an internal 24-base sequence of the 3'-UTR, mRNA carrying the 3'-UTR increased the fluorescence of conjugate $\mathbf{1}$, whereas mRNA lacking the 3'-UTR had no detectable effect. In contrast, in the presence of a 5'-UTR-targeting RTaptamer, both mRNA models increased the fluorescence of conjugate $\mathbf{1}$. Thus, RT-aptamers are able to respond to internal sequences of a long mRNA.

A

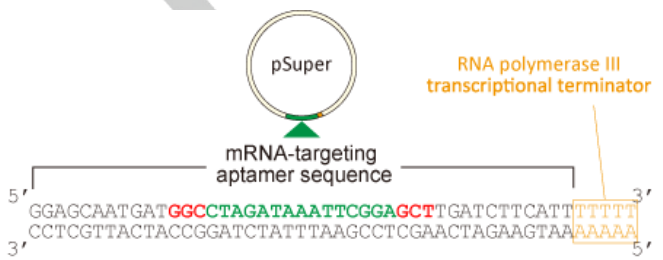

B

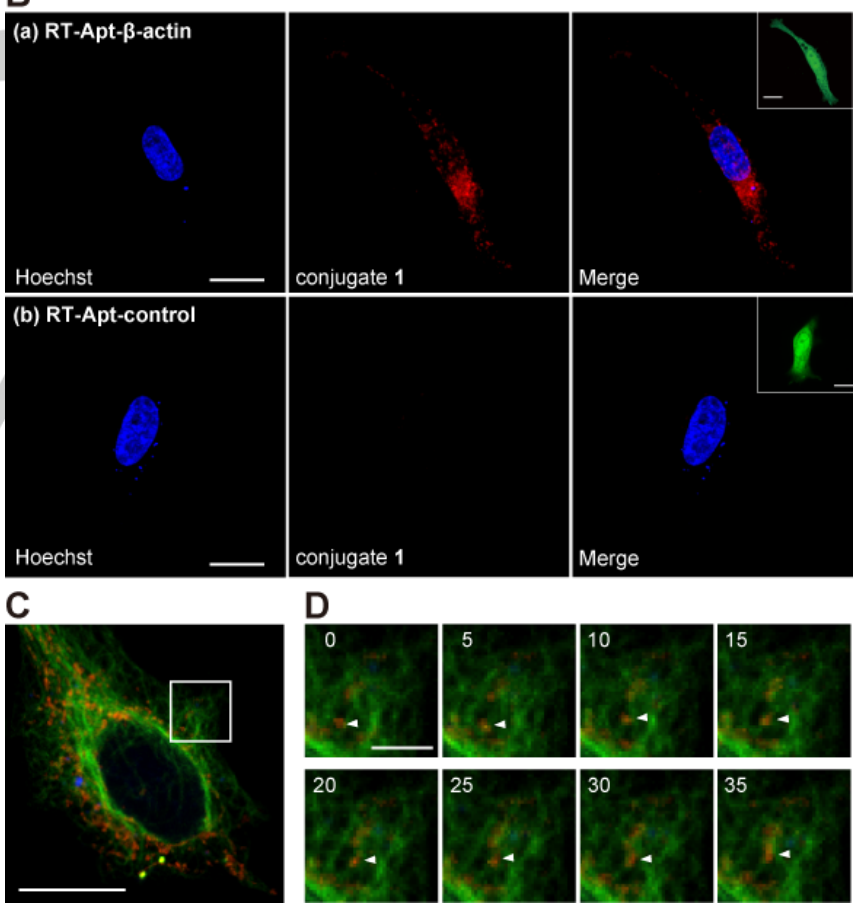

Figure 1. Detection of a target RNA using a combination of a BHQ-fluorophore conjugate with an RNA aptamer. A) Chemical structure of BHQ-fluorophore conjugate 1. B) Design of a BHQ-binding RNA aptamer responsive to a specific sequence of a target RNA. (a) Expected secondary structure of the BHQ aptamer without conjugate 1. The BHQ aptamer comprises the stem structure (red) and the BHQ-recognition loop (green). (b) The BHQ aptamer restores the fluorescence intensities of conjugate 1 by binding to the BHQ-1 moiety of the molecule. (c) Replacement of the stem structure in the BHQ aptamer with two short RNA sequences (blue) that are complementary to a target RNA makes the BHQ-recognition loop flexible, and the resulting RT-aptamer alone cannot bind to conjugate 1 . The uridine mononuculeotides that connect the stem region with the RNA-targeting sequences are shown in orange. (d) An RT-aptamer bound to a target RNA forms a short stem and the stable BHQ-recognition loop structures, and can bind to and restore the fluorescence of conjugate 1 . C)
Figure 2. A) Design and construction of an expression vector encoding RTaptamer. The BHQ-recognition sequence and the transcriptional terminator are shown in green and yellow, respectively. B) Visualization of $\beta$-actin mRNA in a single live HeLa cell expressing (a) RT-Apt- $\beta$-actin or (b) RT-Apt-control. Fluorescent images show Hoechst (blue) and conjugate 1 (red). C) A live-cell image of a single optical plane of $\beta$-actin mRNA granules and microtubules in a HeLa cell expressing RT-Apt- $\beta$-actin. The fluorescent image shows conjugate 1 (red) and TubulinTracker ${ }^{\mathrm{TM}}$ Green (green). D) Time-lapse images of the area denoted by the box in C. Arrowheads denote $\beta$-actin mRNA granules (red) moving along microtubules (green). All times are given in seconds. The insets aptamer. The short hairpin RNA expression vector was used to express an RT- 
in the merged image in B show the fluorescence of GFP, which was used as a marker for expression of the RT-aptamer. Scale bars, $20 \mu \mathrm{m}(\mathrm{B}, \mathrm{C})$ and $5 \mu \mathrm{m}$ (D).

Based on the in vitro results, we applied the new method to endogenous mRNA in living HeLa cells. For the initial trial, we transfected an RNA expression vector encoding an RT-aptamer complementary to an internal 24-base sequence of the $\beta$-actin mRNA (RT-Apt- $\beta$-actin), whose localization has been well studied (Figure 2A and S8A). This target sequence is known to be a highly effective siRNA site ${ }^{[11]}$ and might exhibit high levels of exposure for hybridization ${ }^{[1 a]}$. To validate the expression and interaction of RT-Apt- $\beta$-actin in cells, we purified total mRNAs using an oligo-dT column, twenty-four hours after transfection, and followed the amount of co-purified RT-Apt- $\beta$-actin by real-time quantitative reverse transcription PCR (RT-qPCR) experiments (Supplementary Results and Table S1). The amounts of RTApt- $\beta$-actin were $\sim 18$-fold higher than that of a non-targeting aptamer control (RT-Apt-control), suggesting that RT-Apt- $\beta$-actin is expressed and bound to mRNA in living cells. Moreover, results of RT-qPCR experiments using total RNA indicated that the expression of RT-Apt$\beta$-actin had no detectable effects on the amount of $\beta$-actin mRNA, ruling out the possibility of gene silencing by RT-Apt- $\beta$-actin.
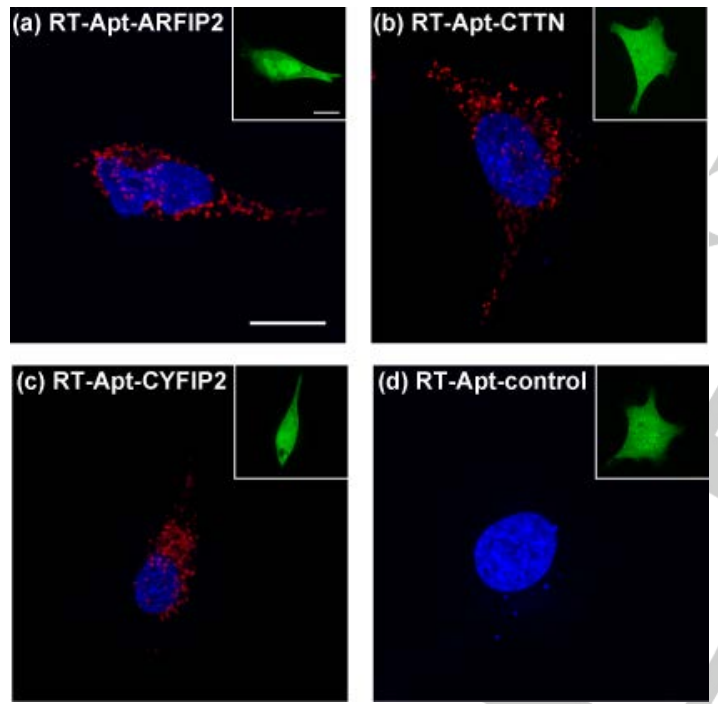

Figure 3. Visualization of the mRNAs of (a) ARFIP2, (b) CTTN, and (c) CYFIP2 in a single live HeLa cell expressing each mRNA-targeting RT-aptamer or (d) RT-Apt-control. Fluorescent images show Hoechst (blue) and conjugate 1 (red) The insets in the merged image show the fluorescence of GFP, which was used as a marker for expression of the RT-aptamer. Scale bar, $20 \mu \mathrm{m}$.

The second step was addition of cell-permeable conjugate $\mathbf{1}$ to the cell culture, allowing the use of fluorescence to detect the location of the $\beta$ actin mRNA/RT-Apt- $\beta$-actin complex. The cells were treated with conjugate 1 twenty-four hours after transfection. Spots of strong fluorescence were observed in the cells expressing RT-Apt- $\beta$-actin, whereas only weak background fluorescence was observed in the cells expressing RT-Apt-control (Figure 2B). Time-lapse images revealed that the fluorescent spots (mRNA granules) were concentrated around the nucleus and moved relatively freely away from the nucleus (Videos S1 and S2). $\beta$-actin mRNA has been demonstrated to be transported along microtubule fibers in cells ${ }^{[12]}$. Co-staining experiments showed that the mRNA granules were co-localized with and moved along microtubule fibers that were stained with TubulinTracker ${ }^{\mathrm{TM}}$ Green (Figures 2C, D and Video S3). The identity of the $\beta$-actin mRNA granules was confirmed independently by fluorescence in situ hybridization (FISH) in fixed cells. The fluorescence signals overlapped those of a $\beta$-actin FISH probe, which hybridizes at a different site from the site targeted by RT-Apt- $\beta$-actin (Figure S8B).

Theoretically, the new RNA-imaging method should allow live-cell imaging of any RNA molecules of interest. To validate this versatility, we prepared a library of RT-aptamer expression vectors that specifically targeted 84 genes encoding cytoskeleton-related proteins (Figure S9). The RT-aptamers were designed to target siRNA sites. Each vector was transfected into HeLa cells, and the cells were analyzed under a confocal microscope in the presence of conjugate $\mathbf{1}$. Ten of the transfected vectors exhibited clear signals, and we focused on the mRNAs of arfaptin-2 (ARFIP2), cortactin (CTTN), and cytoplasmic FMR1-interacting protein 2 (CYFIP2) (Figure 3). The fluorescence signals overlapped in fixed cells with the fluorescence of a FISH probe for each gene, confirming the identity of the RNA (Figure S10). Time-lapse images of living cells revealed dynamics of the mRNA granules, although different genes showed different numbers of granules (Videos S4-S6). The number of the mRNA granules in cells roughly corresponded to the expression levels of mRNAs indicated by RT-qPCR (Figure S11) mRNAs with high expression levels tended to be more visible.

The present study developed a new, versatile and easy-to-use method for live-cell imaging of endogenous RNAs. A common problem in the detection of endogenous mRNAs in living cells is inaccessibility of target sites, making it difficult to design suitable probes ${ }^{[13]}$. Accessibility to mRNA sites is not predictable from their nucleotide sequence information, due to the potential formation of complex secondary and tertiary structures, and tight association with RNA-binding proteins ${ }^{[14]}$. The method reported here is limited by this inaccessibility. We encountered this problem when we designed RT-aptamers for GFP mRNA: RT-aptamers that target 5'-terminal region of 5'-UTR and 3'terminal region of 3'-UTR, which are likely to form a stable secondary structure, failed to fully restore the fluorescence of conjugate $\mathbf{1}$ (Figure S7). However, the new method should be able to overcome this limitation by designing and screening a series of multiple RT-aptamers that target various sites.

Background signals, induced by binding to false or partially complementary targets in cells, are another complication of methods using oligonucleotide-hybridization probes ${ }^{[8]}$, including molecular beacons. Dual FRET linear probes ${ }^{[15]}$ and dual FRET molecular beacons ${ }^{[16]}$ have been developed to improve the signal-to-noise ratio. Our nonFRET method displays relatively high sequence-selectivity and signalto-background ratio, because of the dependence of the signal on a structural transition: when an RT-aptamer hybridizes with false targets, the RT-aptamer fails to form the BHQ-recognition loop structure.

The new method offers advantages: (i) a sequence-selective RTaptamer can easily be designed for a given mRNA target, (ii) RTaptamers can be expressed in cells using basic cell biology techniques, (iii) a small molecule can simply be added to the sample at any time point, and (iv) high stability of the small molecule allows observation over relatively long periods of time. However, the method does require relatively high expression levels of the target mRNAs. Further optimization of the new method might increase sensitivity and permit detection of low-abundance transcripts. For example, signal intensity could be enhanced by employing multiple RT-aptamers that target a 
single RNA transcript. Overall, the present study successfully demonstrates a small molecule-based method that permits spatiotemporal imaging of specific native RNAs in living cells.

Keywords: RNA live-cell imaging $•$ RNA aptamer $\bullet$ fluorescent small molecule $\bullet$ RNA dynamics

a) A. M. Femino, F. S. Fay, K. Fogarty, R. H. Singer, Science 1998, 280, 585-590; b) S. Itzkovitz, A. Lyubimova, I. C. Blat, M. Maynard, J. van Es, J. Lees, T. Jacks, H. Clevers, A. van Oudenaarden, Nat. Cell. Biol. 2012 14, 106-114; c) A. Raj, P. van den Bogaard, S. A. Rifkin, A. van Oudenaarden, S. Tyagi, Nat. Methods 2008, 5, 877-879.

[2] A. R. Buxbaum, B. Wu, R. H. Singer, Science 2014, 343, 419-422.

[3] a) H. Y. Park, H. Lim, Y. J. Yoon, A. Follenzi, C. Nwokafor, M. LopezJones, X. Meng, R. H. Singer, Science 2014, 343, 422-424; b) P. J. Santangelo, A. W. Lifland, P. Curt, Y. Sasaki, G. J. Bassell, M. E. Lindquist, J. E. Crowe, Jr., Nat. Methods 2009, 6, 347-349; c) S. Tyagi, Nat. Methods 2009, 6, 331-338.

[4] a) E. Bertrand, P. Chartrand, M. Schaefer, S. M. Shenoy, R. H. Singer, R. M. Long, Mol. Cell. 1998, 2, 437-445; b) L. Haim, G. Zipor, S. Aronov, J. E. Gerst, Nat. Methods 2007, 4, 409-412; c) T. Lionnet, K. Czaplinski, X. Darzacq, Y. Shav-Tal, A. L. Wells, J. A. Chao, H. Y. Park, V. de Turris, M. Lopez-Jones, R. H. Singer, Nat. Methods 2011, 8, 165-170; d) S. Mili, K. Moissoglu, I. G. Macara, Nature 2008, 453, 115-119; e) Y. Shav-Tal, X. Darzacq, S. M. Shenoy, D. Fusco, S. M. Janicki, D. L. Spector, R. H. Singer, Science 2004, 304, 1797-1800.
[5]

a) J. S. Paige, T. Nguyen-Duc, W. Song, S. R. Jaffrey, Science 2012, 335, 1194; b) W. Song, R. L. Strack, S. R. Jaffrey, Nat. Methods 2013, 10, 873875; c) R. L. Strack, M. D. Disney, S. R. Jaffrey, Nat. Methods 2013, 10, 1219-1224.

[6] S. Tyagi, F. R. Kramer, Nat. Biotech. 1996, 14, 303-308.

[7] a) D. P. Bratu, B. J. Cha, M. M. Mhlanga, F. R. Kramer, S. Tyagi, Proc. Natl. Acad. Sci. USA 2003, 100, 13308-13313; b) D. L. Sokol, X. Zhang, P. Lu, A. M. Gewirtz, Proc. Natl. Acad. Sci. USA 1998, 95, 11538-11543; c) S. Tyagi, O. Alsmadi, Biophys. J. 2004, 87, 4153-4162.

[8] F. Hovelmann, I. Gaspar, S. Loibl, E. A. Ermilov, B. Roder, J. Wengel, A. Ephrussi, O. Seitz, Angew. Chem. Int. Ed. 2014, 53, 11370-11375.

[9] T. Ozawa, Y. Natori, M. Sato, Y. Umezawa, Nat. Methods 2007, 4, 413419.

[10] A. Murata, S. Sato, Y. Kawazoe, M. Uesugi, Chem. Commun. 2011, 47, 4712-4714.

[11] J. Harborth, S. M. Elbashir, K. Bechert, T. Tuschl, K. Weber, J. Cell. Sci. 2001, 114, 4557-4565.

[12] a) D. Fusco, N. Accornero, B. Lavoie, S. M. Shenoy, J. M. Blanchard, R. H. Singer, E. Bertrand, Curr. Biol. 2003, 13, 161-167; b) H. Yoshimura, A. Inaguma, T. Yamada, T. Ozawa, ACS Chem. Biol. 2012, 7, 999-1005.

[13] W. J. Rhee, P. J. Santangelo, H. Jo, G. Bao, Nucl. Acids Res. 2008, 36, e30

[14] S. I. Rudnick, J. Swaminathan, M. Sumaroka, S. Liebhaber, A. M. Gewirtz, Proc. Natl. Acad. Sci. USA 2008, 105, 13787-13792.

[15] A. Tsuji, Y. Sato, M. Hirano, T. Suga, H. Koshimoto, T. Taguchi, S. Ohsuka, Biophys. J. 2001, 81, 501-515.

[16] P. J. Santangelo, B. Nix, A. Tsourkas, G. Bao, Nucl. Acids Res. 2004, 32, e57. 
WILEY-VCH

Table of Contents

\section{COMMUNICATION}

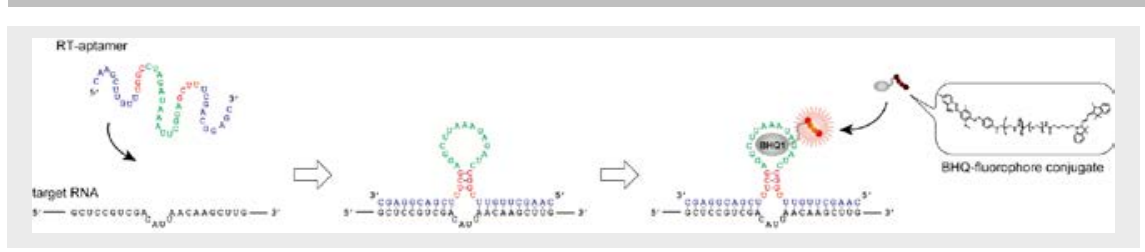

A convenient and versatile method permits spatiotemporal imaging of specific nonengineered RNAs in living cells. The method uses transfection of a plasmid encoding a genespecific RNA aptamer, combined with a cell-permeable synthetic small molecule whose fluorescence is restored only when the RNA aptamer hybridizes with its cognitive mRNA. The method was validated by live-cell imaging of endogenous mRNAs.
Shin-ichi Sato, * Mizuki Watanabe, Yousuke Katsuda, Asako Murata, Dan Ohtan Wang, and Motonari Uesugi*

Page No. XXXX - Page No. XXXX Live-Cell Imaging of Endogenous mRNAs with a Small Molecule 